\title{
A Utilização de Telemedicina para o Aconselhamento Genético em Oncologia
}

doi: https://doi.org/10.32635/2176-9745.RBC.2020v66n1.770

\author{
The Use of Telemedicine for Genetic Counseling in Oncology \\ La Utilización de Telemedicina para la Asesoramiento Genético en Oncología
}

\begin{abstract}
Dione Fernandes Tavares'; Larissa Souza Mario Bueno²; Thiago Rhangel Gomes Teixeira³; Laércio Moreira Cardoso-Júnior; Gabriela Ramos Varrone ${ }^{5}$
\end{abstract}

Resumo

Introduçáo: $\mathrm{O}$ desenvolvimento da medicina com a descoberta do genoma humano revolucionou o conhecimento, o acompanhamento e o tratamento do câncer, proporcionando a utilização de novos métodos de diagnóstico e tratamento para diversos tipos de neoplasias e permitindo o aconselhamento genético. $\mathrm{O}$ aconselhamento genético, embora de difícil acesso, pode vir a permitir aos pacientes com suspeita de câncer hereditário a diminuiçáo dos índices de morbidade e mortalidade por essa doença e proporcionar uma melhora na qualidade de vida dos pacientes. Objetivo: Demonstrar o estado da arte do aconselhamento genético em oncologia, utilizando a ferramenta digital da telemedicina. Método: Foi realizada uma revisão integrativa de literatura, entre dezembro de 2018 e março de 2019, a partir das bases de dados PubMed, SciELO e BIREME, por meio das palavras cadastradas no MeSH e nos Descritores em Ciências da Saúde (DeCS), respectivamente, em inglês, Genetic Counseling AND Telemedicine OR eHealth AND Oncology. Resultados: Foram encontrados 16 artigos; destes, oito foram excluídos. Restaram sete artigos que atendiam aos critérios de inclusão, demonstrando dados sobre a satisfação do paciente para com os serviços de aconselhamento genético voltados para o câncer, assim como suas vantagens e desvantagens. Conclusáo: Embora ainda haja poucos estudos relatando experiências da prática do aconselhamento genético em oncologia, essa prática tem se demonstrado eficiente, de baixo custo e de boa aceitação do paciente e do profissional, suprindo necessidades principalmente nos locais de difícil acesso. Palavras-chave: Aconselhamento Genético; Telemedicina; Oncologia.

\begin{abstract}
Introduction: The development of medicine and the discovery of the human genome revolutionized the knowledge, monitoring and treatment of cancer that favored the utilization of new methods of diagnosis, treatment for several types of neoplasms and genetic counseling. Although difficult to access, genetic counseling may allow the patients with suspected hereditary cancer, to reduce the rates of morbidity and mortality by this disease and ensure improvement in their quality of life. Objective: Demonstrate the state of the art of genetic counseling in oncology using the digital telemedicine tool. Method: An integrative literature review conducted between December 2018 and March 2019, using the PubMed, SciELO and BIREME databases, utilizing the words registered in the MeSH and Health Sciences Descriptors (DeCS), respectively, in English, Genetic Counseling AND Telemedicine OR eHealth AND Oncology. Results: 16 articles were found and of these, eight were excluded. Thus, seven articles that met the inclusion criteria showing data about patient satisfaction with cancer-oriented counseling services, its advantages and disadvantages remained. Conclusion: Although there are few studies addressing the experience of genetic counseling in oncology, this low cost practice has been shown to be efficient and well accepted by the patient and the professional, providing proper responses mainly in places of difficult access.

Key words: Genetic Counseling; Telemedicine; Oncology.
\end{abstract}

Resumen

Introducción: El desarrollo de la medicina con el descubrimiento del genoma humano revolucionó el conocimiento, seguimiento y terapia del cáncer, permitiendo la utilización de nuevas técnicas de diagnóstico y terapias para diferentes tipos de neoplasias, además del asesoramiento genético. El asesoramiento genético, aunque de acceso difícil, puede permitir a los pacientes con sospechas de cáncer hereditario la disminución de los índices de morbilidad y mortalidad para esa enfermedad y proporcionar una mejora en su calidad de vida. Objetivo: Demostrar el estado del arte del asesoramiento genético en oncología utilizando la herramienta digital de la telemedicina. Método: Se realizó una revisión integrativa de literatura entre diciembre de 2018 y marzo de 2019, a partir de las bases de datos PubMed, SciELO y BIREME, por medio de las palabras catastradas en el $\mathrm{MeSH}$ y en el Descriptores de Ciencia de la Salud (DeCS), respectivamente, en inglés Genetic Counseling AND Telemedicine OR eHealth AND Oncology. Resultados: Se han encontrado 16 artículos; de estos, 8 fueron excluidos. Así, quedaron siete artículos que atendían a los criterios de inclusión demostrando datos sobre la satisfacción del paciente hacia con los servicios de asesoramiento genético dirigidos al cáncer, así como sus ventajas y desventajas. Conclusión: Aunque todavía hay pocos estudios relatando experiencias de la práctica del asesoramiento genético en oncología, esta práctica se ha demostrado eficiente, de bajo costo y de buena aceptación del paciente y del profesional, supliendo necesidades principalmente en los lugares de difícil acceso.

Palabras-chave: Asesoramiento Genético; Telemedicina; Oncología.

\footnotetext{
${ }^{1}$ Faculdade de Medicina da Bahia da Universidade Federal da Bahia (FMB/UFBA). Salvador (BA), Brasil. Orcid iD: https://orcid.org/0000-0002-2438-5297

${ }^{2}$ Complexo Hospitalar Universitário Professor Edgard Santos da Maternidade Climério de Oliveira. Centro de Hematologia e Oncologia. Salvador (BA), Brasil. Orcid iD: https://orcid.org/0000-0002-0533-7871

${ }^{3}$ Instituto de Saúde Coletiva da UFBA. Salvador (BA), Brasil. Orcid iD: https://orcid.org/0000-0003-1287-0620

${ }^{4}$ Fundação Estatal Saúde da Família. Salvador (BA), Brasil. Orcid iD: https://orcid.org/0000-0003-0649-1237

${ }^{5}$ FMB/UFBA. Salvador (BA), Brasil. Orcid iD: https://orcid.org/0000-0002-4427-0868

Endereço para correspondência: Dione Fernandes Tavares. Programa de Pós-Graduação em Medicina e Saúde. Hospital Universitário Professor Edgard Santos. UFBA. Rua Doutor Augusto Viana, s/n - Canela. Salvador (BA), Brasil. CEP 40110-060. E-mail: dionefernandestavares@gmail.com
} 


\section{INTRODUÇÃO}

O desenvolvimento da oncogenética é relativamente recente e estima-se que 5\% a $10 \%$ dos casos de câncer têm origem hereditária. Nesses casos, há a possibilidade de medidas preventivas para a redução de risco e detecção precoce com aumento das chances de cura para certos tipos de câncer. A partir de um mapeamento do risco para cada indivíduo, é possível identificar se as chances de desenvolvimento do câncer são elevadas ou não, permitindo-se adotar as medidas necessárias. $\mathrm{O}$ aconselhamento genético é procedimento rotineiro em alguns países desenvolvidos e, nesses países, a mortalidade por diversos tumores tem diminuído satisfatoriamente, podendo este ser um fator a contribuir ${ }^{1}$.

Alguns achados gerais alertam para uma possível causa genética, entre eles, a presença de tumores raros, idade mais jovem que a habitual, vários casos de câncer na família, especialmente em parentes de primeiro e segundo graus, e o histórico de duas ou mais neoplasias em uma mesma pessoa. Em alguns casos, a presença de certas neoplasias combinadas à idade e/ou ao sexo do paciente levam à indicação de avaliaçáo como, por exemplo, câncer de mama antes dos 50 anos ou câncer de mama em homens. Em certos casos, a histologia tumoral por si só é indicação para aconselhamento e exame genético, como é o caso dos carcinomas epiteliais de ovário e do carcinoma de plexo-coroide ${ }^{2}$.

$\mathrm{O}$ aconselhamento genético baseia-se na história pessoal, na história familiar e nos achados específicos de exame físico e resultado de exames de diagnóstico, notadamente os genéticos. O inventário familiar extenso, com levantamento especialmente dos casos de neoplasias benignas, malignas e autismo na família, é fundamental para identificar se há possibilidade de predisposição hereditária a neoplasias malignas ou se há indícios de condição esporádica. Na prática, é montada uma árvore genealógica da família manualmente ou por meio de softwares. Programas de computador podem, por intermédio de algoritmos matemáticos, auxiliar na quantificação do risco para predisposição genética dos pacientes $^{2}$.

Se após o mapeamento houver a suspeita para câncer hereditário, são realizados procedimentos para diagnóstico molecular sempre que disponíveis. O médico oncogeneticista deve saber quais são os genes a serem avaliados e qual a técnica a ser utilizada em cada caso. Só entáo confirmada a presença do defeito no gene que pode favorecer o surgimento do câncer, variante patogênica ou provavelmente patogênica, o paciente recebe as orientaçôes específicas para a prevenção e o rastreamento para neoplasias ${ }^{2}$.
Síndromes genéticas como a de Li Fraumeni, síndrome de Lynch, síndrome do câncer de mama e ovários hereditários, polipose adenomatosa familiar, neoplasias endócrinas múltiplas e a síndrome de Von Hippel Lindau são alguns exemplos de síndromes genéticas familiares que aumentam a chance de câncer e que possuem protocolos de acompanhamento para a adoção de medidas preventivas. Identificada a alteração genética responsável pela síndrome em uma determinada família, são possíveis o diagnóstico precoce e a adoção de medidas preventivas em familiares não afetados ${ }^{2}$.

O diagnóstico quanto à síndrome de predisposição é um ato médico, mas tanto médicos quanto profissionais de algumas outras especialidades podem auxiliar no aconselhamento genético. Em alguns países, existem profissionais formados especificamente para serem aconselhadores genéticos ${ }^{3}$.

A regulamentação da telemedicina no Brasil carece de atualizaçóes, algumas como as contempladas pela revogada Resoluçáo CFM n. ${ }^{\circ}$ 2.227/20184 ${ }^{4}$ Atualmente, ela é regulamentada pela Resolução CFM n. ${ }^{\circ} 1.643 / 2002^{5} \mathrm{e}$ definida como um modelo de prestação de serviços no qual especialistas credenciados prestam cuidados remotamente por meio de "metodologias interativas de comunicação audiovisual e de dados com o objetivo de assistência, educação e pesquisa em Saúde"s. Essa modalidade de atendimento é especialmente importante para clientes de áreas carentes de médicos. Trabalhos realizados anteriormente sugerem que a telemedicina em genética, telegenética, permite fornecer acesso a serviços entre aqueles que enfrentam barreiras geográficas, melhorar a eficiência de custos e atender à crescente demanda por serviços ${ }^{6}$. Essa questáo também foi ressaltada no Processo-Consulta CFM n. ${ }^{\circ}$ 8.732/2009 - PARECER CFM n.o $17 / 2018$, que ressalta que o aconselhamento genético pode e deve ser feito pelos meios de comunicação eletrônicos, tendo o atual perfil da demografia da especialidade e a carência de profissionais especializados em genética médica no Brasil ${ }^{6}$.

Por conta da baixa disponibilidade de profissionais com capacitação na prática do aconselhamento genético em oncologia e do crescente aumento das indicaçóes para avaliaçáo quanto à síndrome de predisposiçáo genética a câncer, o presente trabalho pretende demonstrar o estado da arte do aconselhamento genético em oncologia, utilizando a telemedicina como ferramenta para levar o acesso à informação.

\section{MÉTODO}

Foi realizada uma revisão de literatura integrativa, entre dezembro de 2018 e março de 2019, na qual, 
para o levantamento dos estudos sobre a utilização da telemedicina na prática do aconselhamento genético em oncologia, realizou-se, em primeiro lugar, um estudo de caráter exploratório visando ao dimensionamento do número de trabalhos indexados em diferentes bases de dados, entre as quais, foram consultadas: a) Scientific Electronic Library Online (SciELO); b) Literatura Latino-Americana e do Caribe em Ciências da Saúde (LILACS); e c) U. S. National Library of Medicine (PubMed).

Após consulta aos Descritores em Ciências da Saúde (DeCS), elegeram-se os seguintes descritores "Aconselhamento Genético", "Telemedicina”, "Telessaúde” e "Oncologia" para realização de estudo comparativo nas bases de dados SciELO e LILACS. Após consulta ao Medical Subject Headings (MeSH), selecionaram-se os seguintes descritores "Genetic Counseling", "Telemedicine", "eHealth" e "Oncology" para realização de estudo comparativo nas bases de dados PubMed.

Os critérios de inclusão foram artigos de janeiro de 2009 a outubro de 2018 em português ou inglês; e os critérios de exclusão, revisôes de literatura independente da metodologia aplicada. Em razáo da inexistência de trabalhos nas bases de dados SciELO e LILACS, utilizaram-se apenas trabalhos da base PubMed, que apresentou um total de 16 artigos. Após a aplicação dos critérios de inclusão e exclusão, restaram sete artigos, cujas características estão presentes no Quadro 1 e as vantagens e desvantagens descritas no Quadro 2 dos resultados.

\section{RESULTADOS}

Por meio da busca realizada, foram encontrados 16 artigos, os quais foram selecionados mediante critérios de inclusão e exclusão do estudo. Dessa forma, foram incluídos sete artigos.

\section{DISCUSSÃO}

O uso de telegenética é cada vez mais visto como uma alternativa de acesso a informaçóes de saúde em áreas remotas ${ }^{7}$. Um estudo prévio de telegenética-piloto envolvendo pacientes do Maine, aconselhados para várias condiçôes genéticas, incluindo a suscetibilidade ao câncer, demonstrou um impacto positivo na tomada de decisão médica entre os estudados, bem como altos níveis de confiança nos provedores. $\mathrm{O}$ estudo concluiu que ao mover a informação, ao invés do paciente, a telemedicina promete melhorar os cuidados de saúde, enquanto desmonta a barreira de onde e quando os serviços genéticos não são fornecidos ${ }^{8}$.

Os trabalhos avaliados demonstraram a satisfação do paciente para com os serviços de aconselhamento genético voltados para o câncer, pois muitas vantagens como a acessibilidade a esse tipo de serviço e a possibilidade de atendimento sem deslocamento foram supridas. De maneira geral, foram observadas poucas desvantagens já esperadas nos serviços telemedicina como a preocupação com o risco de violação de confidencialidade de suas informações de saúde ${ }^{9-11}$. Questóes de segurança e privacidade com comunicação eletrônica foram as desvantagens mais comumente relatadas, o que também foi encontrado em outras pesquisas que utilizam tecnologias de informação e comunicação em saúde ${ }^{12,13}$.

Hilgart et al. ${ }^{14}$ demonstraram que, embora houvesse a preferência dos entrevistados pelo aconselhamento

Quadro 1. Características dos estudos analisados

\begin{tabular}{|c|c|c|c|c|}
\hline $\begin{array}{c}\text { Autor/ano } \\
\text { Bradbury et } \\
\text { al., } 2016\end{array}$ & 98 & $\begin{array}{c}\text { Estados Unidos da } \\
\text { América }\end{array}$ & Colorretal, mama e ovário & Aconselhamento \\
\hline $\begin{array}{c}\text { Buchanan et } \\
\text { al., } 2015\end{array}$ & 162 & $\begin{array}{c}\text { Estados Unidos da } \\
\text { América }\end{array}$ & $\begin{array}{c}\text { Colorretal, mama, ovário, outros/ } \\
\text { múltiplo }\end{array}$ & Pré e pós-teste \\
\hline $\begin{array}{c}\text { Zilliacus et } \\
\text { al., } 2010\end{array}$ & 18 & Austrália & Mama e ovário & Pós-teste \\
\hline $\begin{array}{c}\text { Hilgart et al., } \\
2012\end{array}$ & 225 & País de Gales & Mama/ovário, colorretal, estômago e & outros \\
\hline $\begin{array}{c}\text { Zilliacus et } \\
\text { al., 2010 }\end{array}$ & 15 & Austrália & Mama e ovário & -- \\
\hline $\begin{array}{c}\text { McDonald et } \\
\text { al., 2014 }\end{array}$ & 149 & $\begin{array}{c}\text { Estados Unidos da } \\
\text { América }\end{array}$ & Mama e ovário & Pré-teste \\
\hline $\begin{array}{c}\text { Zilliacus et } \\
\text { al., 2011 }\end{array}$ & 195 & Austrália & Mama e ovário & Pré e pós-teste \\
\hline
\end{tabular}


Quadro 2. Características da teleconsulta e vantagens e desvantagens do aconselhamento genético utilizando a telemedicina

\begin{tabular}{|c|c|c|c|}
\hline Autor/ano & Teleconsulta & Vantagens & Desvantagens \\
\hline $\begin{array}{l}\text { Bradbury et } \\
\text { al., } 2016\end{array}$ & $\begin{array}{l}\text { A consulta virtual foi realizada com um } \\
\text { conselheiro genético da Universidade da } \\
\text { Pensilvânia. No aconselhamento pré-teste } \\
\text { com apoio da equipe clínica local e suporte } \\
\text { técnico. No aconselhamento pós-teste, } \\
\text { 63\% dos pacientes que necessitavam desse } \\
\text { aconselhamento, realizaram na presença } \\
\text { de um médico local, os outros pacientes } \\
\text { tiveram consulta de seguimento }\end{array}$ & $\begin{array}{l}\text { Baixo custo, sem } \\
\text { necessidade de longas } \\
\text { viagens a grandes centros, } \\
\text { possibilidade agendamento } \\
\text { do aconselhamento em } \\
\text { paralelo com uma consulta } \\
\text { presencial com o médico } \\
\text { local que acompanha o } \\
\text { paciente }\end{array}$ & $\begin{array}{l}\text { Dificuldades } \\
\text { técnicas. Dois } \\
\text { participantes } \\
\text { sentiram falta de } \\
\text { apoio presencial } \\
\text { do conselheiro }\end{array}$ \\
\hline $\begin{array}{l}\text { Buchanan et } \\
\text { al., } 2015\end{array}$ & $\begin{array}{l}\text { Um único conselheiro da Universidade } \\
\text { Duke atendeu a todos os pacientes, } \\
\text { via telemedicina da universidade para } \\
\text { a clínica regional onde o paciente } \\
\text { era acompanhado. O conselheiro e o } \\
\text { paciente se comunicaram em tempo } \\
\text { real em uma tela e visualizaram os } \\
\text { documentos comumente usados durante o } \\
\text { aconselhamento em uma segunda tela }\end{array}$ & $\begin{array}{l}\text { Menor custo por paciente } \\
\text { por meio da telemedicina } \\
\text { (\$ 106) em comparação } \\
\text { com aconselhamento } \\
\text { presencial (\$244) }\end{array}$ & $\begin{array}{l}\text { Uma taxa de } 32 \% \\
\text { dos participantes } \\
\text { listou que seria } \\
\text { uma desvantagem } \\
\text { não ter contato } \\
\text { presencial com o } \\
\text { conselheiro }\end{array}$ \\
\hline $\begin{array}{l}\text { Zilliacus et } \\
\text { al., } 2010\end{array}$ & $\begin{array}{l}\text { Neste estudo, } 18 \text { participantes tiveram } \\
\text { aconselhamento genético a partir } \\
\text { da telemedicina utilizando o sistema } \\
\text { Tandberg }^{\mathrm{TM}} \text {. Os tamanhos dos monitores } \\
\text { eram, em média, telas de } 42 \text { polegadas. } \\
\text { As câmeras permitiam um campo de visão } \\
\text { horizontal de } 72^{\circ} \text { e um vertical de } 43,5^{\circ} \text {. } \\
\text { As câmeras utilizadas têm a capacidade } \\
\text { de acomodar três pessoas (conselheiro } \\
\text { genético, paciente e pessoa de apoio) e } \\
\text { também têm a capacidade de ampliação } \\
\text { para capturar a parte superior do corpo e } \\
\text { o rosto de uma pessoa (geralmente clínico } \\
\text { genético) para aumentar a expressão facial } \\
\text { e tornar a consulta mais acolhedora }\end{array}$ & $\begin{array}{l}\text { Redução das viagens e } \\
\text { custos associados }\end{array}$ & $\begin{array}{l}\text { Falta de apoio } \\
\text { emocional }\end{array}$ \\
\hline $\begin{array}{l}\text { Hilgart et al., } \\
2012\end{array}$ & $\begin{array}{l}\text { Pesquisadores por meio do banco de } \\
\text { dados de pesquisas prévias convidaram } \\
\text { pacientes e funcionários para participarem } \\
\text { de uma pesquisa, a fim de avaliar a } \\
\text { aceitabilidade e a viabilidade do uso da } \\
\text { telemedicina nos serviços de genética do } \\
\text { câncer. Muitos aspectos da telemedicina } \\
\text { no aconselhamento genético propostos } \\
\text { na pesquisa foram altamente aceitáveis } \\
\text { para os pacientes, incluindo uma versão } \\
\text { eletrônica do questionário de história } \\
\text { da família, um recurso de e-mail para } \\
\text { consultas genéticas do câncer e um auxílio } \\
\text { à decisão computadorizada }\end{array}$ & $\begin{array}{l}\text { Facilidade do acesso à } \\
\text { informação via e-mail e } \\
\text { outros métodos eletrônicos } \\
\text { sem a necessidade de } \\
\text { viajar para um centro } \\
\text { referência }\end{array}$ & $\begin{array}{l}\text { Impessoalidade e } \\
\text { falta de contato } \\
\text { humano por } \\
\text { causa do uso } \\
\text { dos métodos } \\
\text { eletrônicos }\end{array}$ \\
\hline $\begin{array}{l}\text { Zilliacus et } \\
\text { al., } 2010\end{array}$ & $\begin{array}{l}\text { Este trabalho pretendia avaliar a satisfação } \\
\text { e experiência dos profissionais que } \\
\text { realizaram aconselhamento genético } \\
\text { por meio da telemedicina em pacientes } \\
\text { oncológicos, dessa forma, entrevistas } \\
\text { semiestruturadas foram realizadas com } \\
\text { médicos genéticos prestando serviços em } \\
\text { New South Wales, Austrália. As entrevistas } \\
\text { exploraram experiências, objetivos } \\
\text { percebidos do serviço, satisfação e } \\
\text { vantagens e desvantagens da tecnologia }\end{array}$ & $\begin{array}{l}\text { Uma maior eficiência foi } \\
\text { verificada, minimização } \\
\text { da viagens e custos } \\
\text { associados, maior acesso } \\
\text { às áreas rurais }\end{array}$ & $\begin{array}{c}\text { Ausência da } \\
\text { criação de vínculo }\end{array}$ \\
\hline
\end{tabular}


Quadro 2. continuação

\begin{tabular}{|c|c|c|c|}
\hline Autor/ano & Teleconsulta & Vantagens & Desvantagens \\
\hline $\begin{array}{c}\text { McDonald et } \\
\text { al., } 2014\end{array}$ & $\begin{array}{l}\text { A consulta de aconselhamento genético } \\
\text { foi realizada por meio da telemedicina } \\
\text { e avaliada pela aplicação de um } \\
\text { questionário composto por } 27 \text { perguntas, } \\
\text { que avaliam dados demográficos, histórico } \\
\text { pessoal e familiar de câncer, assim como } \\
\text { a avaliação de risco de suscetibilidade } \\
\text { ao câncer, conhecimento sobre testes } \\
\text { genéticos em oncologia, aceitabilidade de } \\
\text { características que refletem modelos de } \\
\text { prestação de serviços de aconselhamento } \\
\text { genético e a qualificação e avaliação do } \\
\text { aconselhamento genético }\end{array}$ & $\begin{array}{c}\text { Acessibilidade de } \\
\text { ter contato com um } \\
\text { profissional qualificado } \\
\text { atendendo e aconselhando } \\
\text { a distância }\end{array}$ & -- \\
\hline $\begin{array}{c}\text { Zilliacus et } \\
\text { al., } 2011\end{array}$ & $\begin{array}{l}\text { As consultas de aconselhamento genético } \\
\text { envolveram um médico geneticista via } \\
\text { telemedicina, além de um conselheiro } \\
\text { genético local presente com o paciente } \\
\text { em sua cidade de origem em indivíduos } \\
\text { com câncer de mama e ovário hereditário, } \\
\text { onde um grupo recebeu aconselhamento } \\
\text { virtual e outro presencial. As participantes } \\
\text { preencheram questionários autoaplicáveis } \\
\text { antes e um mês após o aconselhamento } \\
\text { genético, demonstrando as expectativas e } \\
\text { avaliando o serviço }\end{array}$ & $\begin{array}{c}\text { O atendimento via } \\
\text { telemedicina teve um } \\
\text { desempenho melhor do } \\
\text { que o aconselhamento } \\
\text { presencial em atender às } \\
\text { expectativas }\end{array}$ & -- \\
\hline
\end{tabular}

presencial, a telemedicina poderia beneficiar as pessoas que estejam impossibilitadas de viajar e que apresentem pouca acessibilidade em virtude do local de residência tanto pela distância quanto por meios de transporte ${ }^{14}$.

Quando comparada a prática do aconselhamento virtual versus o aconselhamento presencial, um estudo randomizado de telegenética descobriu que os serviços de telemedicina custam menos do que o aconselhamento presencial, ainda associado com a alta satisfação entre os clientes, sendo uma alternativa viável e financeiramente melhor para os sistemas de saúde, além de levar acesso à informação genética a lugares remotos ${ }^{10,15}$.

Em relação aos aspectos biopsicossociais envolvidos com o aconselhamento genético e o próprio diagnóstico de câncer, Zilliacus et al. ${ }^{11}$ não encontraram diferença estatisticamente significante nos quesitos ansiedade generalizada, depressão, percepção de empatia do clínico genético e percepção da empatia do conselheiro genético quando comparado o aconselhamento genético virtual versus o presencial ${ }^{11}$.

No País de Gales, foi relatado o caso de uma mulher com diagnóstico recente de câncer em que a telemedicina não era capaz de suprir suas necessidades de apoio psicossocial, necessidades essas que podem ser explicadas pela fase de aceitação da doença vivenciada pela paciente frente ao diagnóstico recente ${ }^{14}$.
No que se refere às expectativas dos pacientes, em um trabalho norte-americano, o aconselhamento genético em oncologia teve desempenho significativamente melhor do que o aconselhamento presencial para atender às expectativas dos pacientes, podendo também se justificar pelo fato de ser algo novo e desconhecido, gerando surpresas aos entrevistados ${ }^{16}$.

Em relação ao lado profissional do aconselhamento genético, nos Estados Unidos da América, percebeu-se que, quando a consulta era realizada com a presença do conselheiro genético, do médico e do paciente, garantindo uma coparticipaçáo, ocorreu um alto nível de troca de informaçóes, oferecendo uma estrutura útil para o aconselhamento genético em telemedicina, papéis complementares entre os profissionais e prestaçáo de serviços eficientes ${ }^{17}$.

Notadamente, a telemedicina, por meio de videoconferência, oferece um modelo alternativo de prestação de serviços de aconselhamento genético para câncer hereditário em populaçôes rurais e de difícil acesso geográfico. Esse método permite que os médicos geneticistas permaneçam em hospital de base vinculando-se aos pacientes por videoconferência para aconselhamento genético. A telemedicina por videoconferência está sendo utilizada com sucesso em outras áreas médicas, como psiquiatria $^{18}$, dermatologia ${ }^{19}$, pediatria ${ }^{20}$ e oncologia ${ }^{21}$. 


\section{CONCLUSÃO}

Com a aumento das indicações de testes genéticos para investigação de predisposição hereditária ao câncer, há uma necessidade crescente de fornecer acesso de qualidade ao aconselhamento genético para garantir a equidade do acesso em diferentes regiôes geográficas. A videoconferência parece ser uma tecnologia alternativa viável.

Embora ainda haja poucos estudos relatando experiências da prática do aconselhamento genético em oncologia, em alguns países, essa modalidade de atendimento tem se demonstrado eficiente, de baixo custo e com boa aceitaçáo do especialista e do paciente, suprindo necessidades principalmente nos locais de difícil acesso.

O modelo de aconselhamento genético virtual pode ser uma ferramenta importante tanto em países subdesenvolvidos e em desenvolvimento, onde há dificuldade no acesso aos serviços de saúde. Sempre levando em consideração que a tecnologia é uma ferramenta de auxílio em casos específicos e em hipótese alguma substitui o trabalho do profissional médico.

\section{CONTRIBUIÇÕES}

Os autores contribuíram igualmente em todas as etapas do estudo e aprovaram a versão final a ser publicada.

\section{DECLARAÇÃO DE CONFLITO DE INTERESSES}

Nada a declarar.

\section{FONTES DE FINANCIAMENTO}

Coordenação de Aperfeiçoamento de Pessoal de Nível Superior (Capes).

\section{REFERÊNCIAS}

1. Foulkes WD. Inherited susceptibility to common cancers. N Engl J Med. 2008;359(20):2143-53. doi: http:// dx.doi.org/10.1056/NEJMra0802968

2. Lindor NM, McMaster ML, Lindor CJ, et al. Concise handbook of familial cancer susceptibility syndromes second edition. J Natl Cancer Inst Monogr. 2008;38:193. doi: http://dx.doi.org/10.1093/jncimonographs/ $\operatorname{lgn} 001$

3. Douma KFL, Smets EMA, Allain DC. Non-genetic health professionals' attitude towards, knowledge of and skills in discussing and ordering genetic testing for hereditary cancer. Fam Cancer. 2016;15(2):341-50. doi: http://dx.doi.org/10.1007/s10689-015-9852-6

4. Conselho Federal de Medicina (BR). Resolução CFM no 2.227/2018 [Internet]. Diário Oficial da União, Brasília,
DF; 2019 fev. 6. Seção I, p. 58. [acesso 2019 maio 20]. Disponível em: https://portal.cfm.org.br/images/PDF/ resolucao222718.pdf

5. Conselho Federal de Medicina (BR). Resolução CFM n ${ }^{\circ}$ 1.643/2002 [Internet]. Diário Oficial da União, Brasília, DF; 2002 ago. 26. Seção I, p. 205. [acesso 2019 maio 20]. Disponível em: http://www.portalmedico.org.br/ resolucoes/CFM/2002/1643_2002.pdf

6. Conselho Federal de Medicina (BR). Parecer CFM no 17/2018 [Internet]. Brasília, DF: CFM; 2017 [acesso 2019 maio 20]. Disponível em: https://sistemas.cfm.org. br/normas/arquivos/pareceres/BR/2018/17_2018.pdf

7. Hilgart JS, Hayward JA, Coles B, et al. Telegenetics: a systematic review of telemedicine in genetics services. Genet Med. 2012;14(9):765-76. doi: http://dx.doi. org/10.1038/gim.2012.40

8. Lea DH, Johnson JL, Ellingwood S, et al. Telegenetics in Maine: Successful clinical and educational service delivery model developed from a 3-year pilot project. Genet Med. 2005;7(1):21-7. doi: http://dx.doi. org/10.1097/01.gim.0000151150.20570.e7

9. Bradbury A, Patrick-Miller L, Harris D, et al. Utilizing remote real-time videoconferencing to expand access to cancer genetic services in community practices: a multicenter feasibility study. J Med Internet Res. 2016;18(2):e23. doi: http://dx.doi.org/10.2196/ jmir.4564

10. Buchanan AH, Datta SK, Skinner CS, et al. Randomized trial of telegenetics vs. in-person cancer genetic counseling: cost, patient satisfaction and attendance. J Genet Couns. 2015;24(6):961-70. doi: http://dx.doi. org/10.1007/s10897-015-9836-6

11.Zilliacus EM, Meiser B, Lobb EA, et al. Women's experience of telehealth cancer genetic counseling. J Genet Couns. 2010;19(5):463-72. doi: http://dx.doi. org/10.1007/s10897-010-9301-5

12. Simon C, Acheson L, Burant C, et al. Patient interest in recording family histories of cancer via the Internet. Genet Med. 2008;10(12):895-902. doi: http://dx.doi. org/10.1097/GIM.0b013e31818de708

13. Grayston J, Fairhurst K, McKinstry B. Using new technologies to deliver test results in primary care: structured interview study of patients' views. Prim Health Care Res Dev. 2010;11(2):142-54. doi: https:// doi.org/10.1017/S146342360999034X

14. Hilgart J, Hayward JA, Iredale R. E-genetics: exploring the acceptability and feasibility of using technology in cancer genetics services. Clin Genet. 2012;81(6):514-20. doi: http://dx.doi.org/10.1111/j.1399-0004.2011.01813.x

15. Zilliacus E, Meiser B, Lobb E, et al. The virtual consultation: practitioners' experiences of genetic counseling by videoconferencing in Australia. Telemed J E Health. 2010;16(3):350-7. doi: http://dx.doi. org/10.1089/tmj.2009.0108 
16. McDonald E, Lamb A, Grillo B, et al. Acceptability of telemedicine and other cancer genetic counseling models of service delivery in geographically remote settings. J Genet Couns. 2014;23(2):221-8. doi: http://dx.doi. org/10.1007/s10897-013-9652-9

17. Zilliacus EM, Meiser B, Lobb EA, et al. Are videoconferenced consultations as effective as face-toface consultations for hereditary breast and ovarian cancer genetic counseling? Genet Med. 2011;13(11):933-41. doi: http://dx.doi.org/10.1097/GIM.0b013e3182217a19

18. Bishop JE, O’Reilly RL, Maddox K, et al. Client satisfaction in a feasibility study comparing faceto-face interviews with telepsychiatry. J Telemed Telecare. 2002;8(4):217-21. doi: http://dx.doi. org/10.1258/135763302320272185

19. Collins K, Walters S, Bowns I. Patient satisfaction with teledermatology: quantitative and qualitative results from a randomized controlled trial. J Telemed Telecare. 2004;10(1):29-33. doi: http://doi. org/10.1258/135763304322764167

20. Connors HR. Telehealth technologies enhance children's health care. J Prof Nurs. 2002;18(6):311-2. doi: https:// doi.org/10.1053/jpnu.2002.130035

21. Mair F, Whitten P. Systematic review of studies of patient satisfaction with telemedicine. BMJ. 2000;320(7248):1517-20. doi: http://dx.doi. org/10.1136/bmj.320.7248.1517 